\title{
Touch Imprint Cytology Of Renal Tumors: Report Of Three Cases
}

\author{
R.M.Potekar and Ambica C*
}

Department of pathology, BLDEU’S Shri B. M. Patil Medical College, Hospital and Research centre, Vijayapur,Karnataka,India.

\begin{abstract}
Imprint is a touch preparation in which the cut surface of the tissue is touched gently on a glass slide which leaves behind its impression in the form of cells. Touch imprint cytology is an important diagnostic tool in surgical oncology. It is reliable and economical providing excellent cellular details. The simplicity, speed and cost effectiveness along with the ability to maximize cell recovery even from a tiny tissue makes imprint cytology a valuable resource in diagnostic medicine. Renal tumors have distinct cytological features that facilitate diagnosis on touch imprint cytology. In the present study, correlation of imprint cytology with histopathological diagnosis was done in three cases of renal tumors.
\end{abstract}

Keywords: Touch Imprint Cytology, Renal Cell Carcinoma, Wilms Tumor, Oncocytoma

\section{Introduction}

The outcome of any medical care is ultimately dependent on rapid and timely diagnosis for the effective management. ${ }^{[1]}$ Touch imprint cytology (TIC) is on the rise in diagnostic cytology because of its main advantage to differentiate between benign and malignant lesions in a shorter time span. ${ }^{[1]}$ Although, histopathological diagnosis is considered gold standard to establish definitive diagnosis, TIC can be considered a diagnostic approach especially during intraoperative period to arrive at a rapid diagnosis, thereby helping the surgeon in planning the management. ${ }^{[1,2,3]}$ The diagnostic accuracy of TIC is comparable to frozen section with an added advantage of being economical and the access to be done in a low facility set-up. ${ }^{[2,4]}$ Here, we present imprint cytology of renal cell carcinoma and Wilms tumor with histopathological correlation in three cases.

\section{Case Reports}

CASE 1A 45 year female presented with hematuria, burning micturition, pain and mass per abdomen since 8 days. On per abdominal examination, a right lumbar mass was noted measuring 10x $8 \mathrm{~cm}$.After a thorough pre operative workup, a right nephroureterectomy with paracacval dissection was performed.Grossly, the specimen measured $17 \times 15 \times 5 \mathrm{~cm}$ with attached ureter measuring $9 \mathrm{~cm}$. On cut surface- a solid fleshy growth was noted measuring $12 \times 10 \mathrm{~cm}$ with areas of hemorrhage and cystic change. Compressed renal tissue noted at foci.

Imprint Cytology: Smears were highly cellular and cells were arranged in loose clusters and singly scattered. Individual cells were large round to polygonal having round to oval nuclei with prominent nucleoli and abundant finely granular eosinophilic to vacuolated cytoplasm
(Figure 1). Few spindle shaped cell clusters were also noted with elongated nuclei, inconspicuous nucleoli and loose chromatin. Cytoplasm was moderate in amount(Figure 1inset). Background showed foamy macrophages. Based on these findings,differential diagnosis of clear cell renal cell carcinoma and Chromophobe renal cell carcinoma (ChRCC) were given.

Histopathology: Tumor tissue was predominantly arranged in diffuse sheets with focal trabeculae, papillary and glandular pattern. Individual cells were large round to polygonal having round vesicular nuclei, prominent nucleoli and abundant clear to granular cytoplasm, suggestive of clear cell RCC. Cystically dilated spaces lined by tumor cells and filled with RBCs and cyst macrophages were also noted (Figure 2).

CASE 2: A 40 year male complained of pain abdomen since 8 days. On examination- a right lumbar mass was noted measuring $7 x 6 \mathrm{~cm}$. Ultrasonography and Computed Tomography suggested neoplastic lesion and cystic RCC respectively.Right nephrectomy was performed. Grossly, the kidney measured $14 \times 9 \times 6 \mathrm{~cm}$ and was capsulated.Cut surface showed an encapsulated, solid, gray white mass in the lower pole which measured $8 \times 7 \times 5.5 \mathrm{~cm}$ with large areas of hemorrhage and necrosis.

Imprint Cytology: Smears were moderately cellular with many atypical cells arranged in monolayered sheets and singly scattered. These cells were round to polygonal with a round to oval eccentric nuclei. Few cells showed irregular nuclear membrane. Cytoplasm was abundant granular eosinophilic to flocculent with a distinct cell borders. Background showed neutrophilic inflammatory infiltrate. 
Based on these findings, differential diagnosis of $\mathrm{ChRCC}$ and oncocytoma was given on imprint cytology(Figure 3).

Histopathology: Tumor tissue was arranged in diffuse sheets. Individual cells were round to polygonal with small round, slightly irregular hyperchromatic nucleus, inconspicuous nucleoli and abundant amount of eosinophilic granular cytoplasm suggestive of ChRCC. Large areas of necrosis was also noted (Figure 4). Special staining by Hale's colloidal iron showed positivity further confirming ChRCC (Figure 5).

CASE 3: A 3 year girl complained of pain and distension of abdomen and hematuria since 3 months. Ultrasonography suggested Wilms tumor and a right radical nephroureterectomy was performed. Grossly, the specimen measured $13 \times 10 \times 8 \mathrm{~cm}$ with lobulated appearance and congested surface. Cut section was gray white with areas of hemorrhage.

Imprint Cytology: High cellular imprint smears showed predominantly blastemal component with cells arranged in small clusters and scattered singly. Individual cells were small, round with round to oval hyperchromatic nuclei, fine granular chromatin, inconspicuous nucleoli and scant, fragile cytoplasm. At foci few spindle shaped cells were also noted(Figure 6).

Histopathology: Triphasic pattern of cells were noted comprising of blastemal, epithelial and mesenchymal stromal components. Blastemal cells were small, round to oval with dark staining nucleus and scant amount of cytoplasm. Epithelial component was predominantly

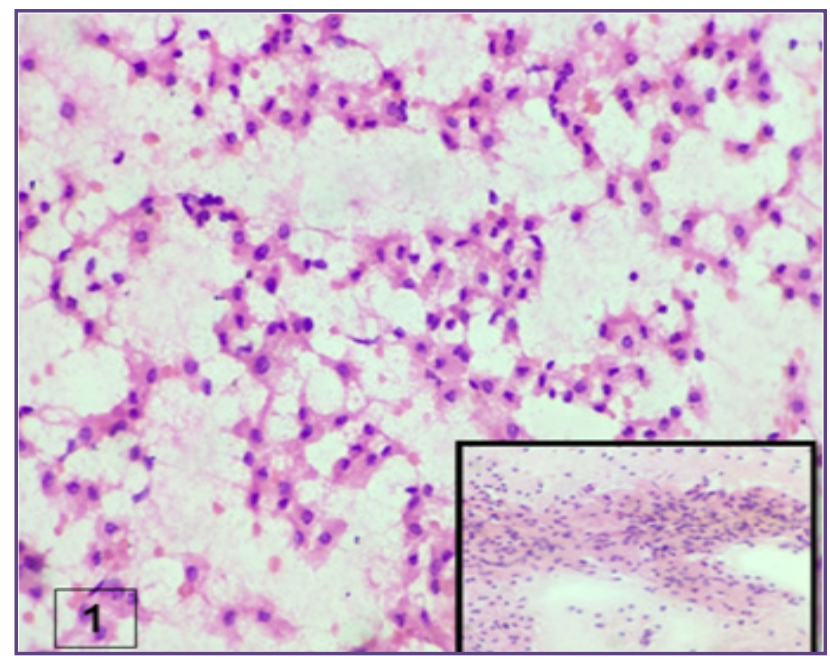

Fig. 1: Microphotograph of imprint smear showing round to oval cells with abundant granular eosinophilic cytoplasm (H\&E 20X). Inset showing spindle shaped cells (H\&E 10X). arranged in tubules and cysts comprised of cuboidal to columnar cells with round to elongated nucleus and moderate amount of eosinophilic cytoplasm. Mesenchymal component showed many spindle shaped cells.

\section{Discussion}

Renal tumors have distinctive cytological features that facilitate specific diagnosis on imprint smears. Renal cell carcinoma is the most common tumor of the kidney comprising about $80-90 \%$ of primary malignant renal neoplasm in adults. ${ }^{[5]}$ The most common histological subtype of RCC is the clear cell or conventional type RCC accounting for $70-80 \%{ }^{[5]} \mathrm{ChRCC}$ is much less common (about $5 \%$ ) as compared to clear cell RCC. ${ }^{[6,7]} \mathrm{ChRCC}$ is unique in having distinct morphological, histological, cytogentic and ultrastructural properties. ${ }^{[6,8]}$ On imprint cytology, ChRCC show highly cellular smears with distinct cell borders and finely granular to transparent cytoplasm. The key feature which helps in diagnosis is the presence of distinct perinuclear halo and slight irregular to wrinkled nuclei. However, in the present cases (Case 1\&2), perinuclear halo and wrinkled nuclei were not prominent, leading to confusion with other diagnosis.

The main differential diagnoses of ChRCC on cytology include renal oncocytoma and clear cell RCC with granular cells. Differentiation of ChRCC from oncocytoma is not only challenging but also important in further management. Renal oncocytoma is a benign tumor and shows monotonous cell population on cytology. The cells have uniform nuclei, homogenous granular cytoplasm without reticulated clearing or vacuolization and without having well defined

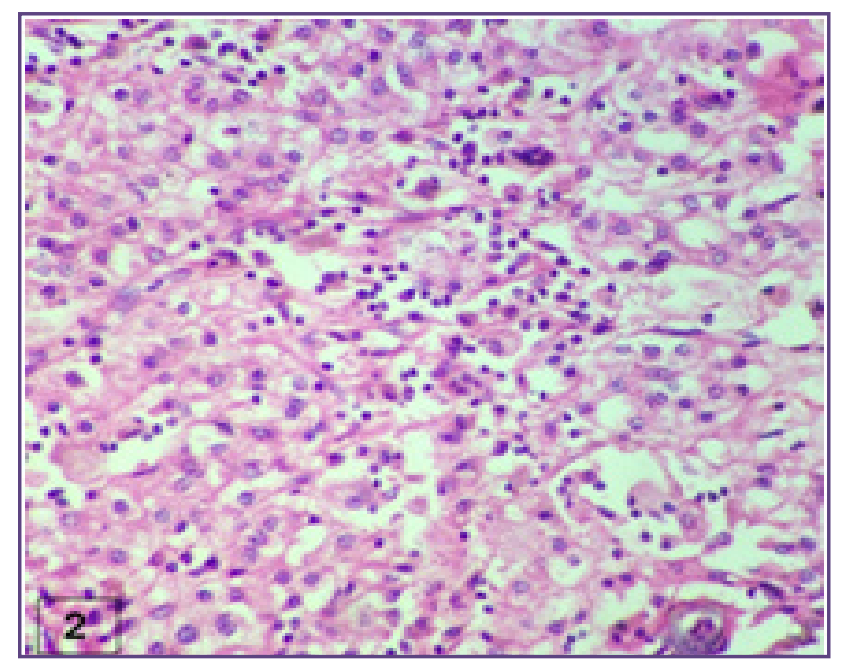

Fig. 2: Microphotograph showing round to polygonal cells with vesicular nuclei, prominent nucleoli and abundant clear to granular eosinophilc cytoplasm (H\&E 200X) 


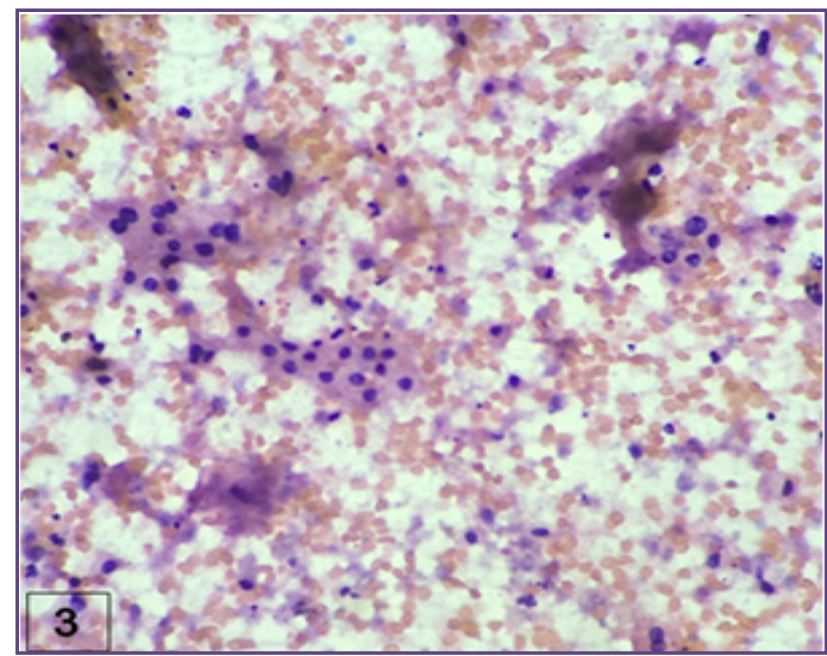

Fig. 3: Microphotograph of imprint smear showing round to oval cells having eccentric nucleus and granular eosinophilic to flocculent cytoplasm in a necrotic background (Giemsa 200X).

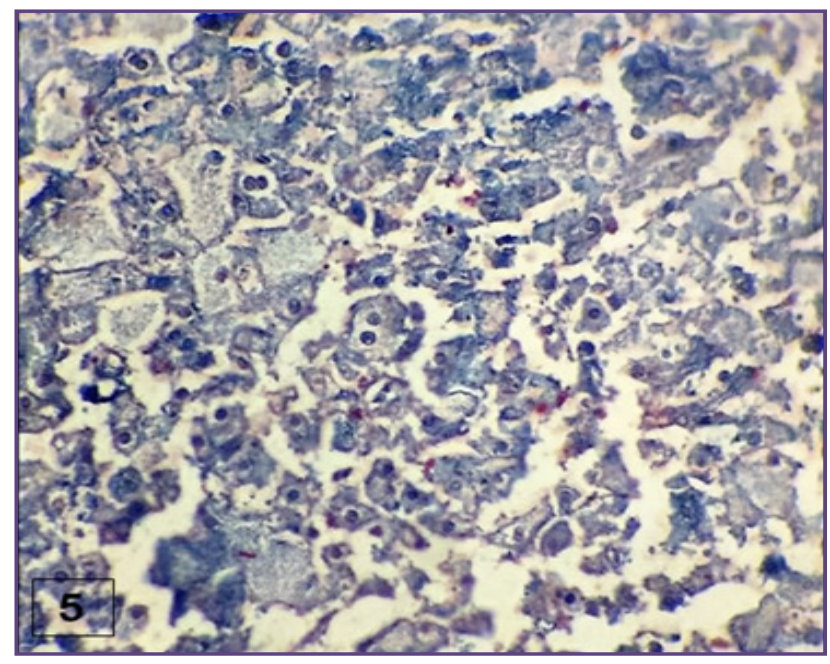

Fig. 5: Microphotograph showing deep blue granules in the cytoplasm (Hale's colloidal iron 200X).

cell borders as compared to ChRCC. ${ }^{[6-8]}$ Special staining by Hale's colloidal iron helps to distinguish the two by being strongly positive in ChRCC and negative in oncocytoma. ${ }^{[6]}$ Immunohistochemistry by vimentin and cytokeratin helps in differentiation between ChRCC and oncytoma. Oncocytoma is positive for cytokeratin and negative for vimentin whereas $\mathrm{ChRCC}$ is positive for both the markers. ${ }^{[6,8]}$ The prognosis of ChRCC falls between oncocytoma and clear cell RCC and usually is managed by simple or partial nephrectomy. Therefore, a correct cytological diagnosis aids in appropriate management. Distinguishing ChRCC from clear cell RCC with granular cells is often difficult. Clear cell RCC is associated with dirty, bubbly background containing foamy macrophages whereas ChRCC usually

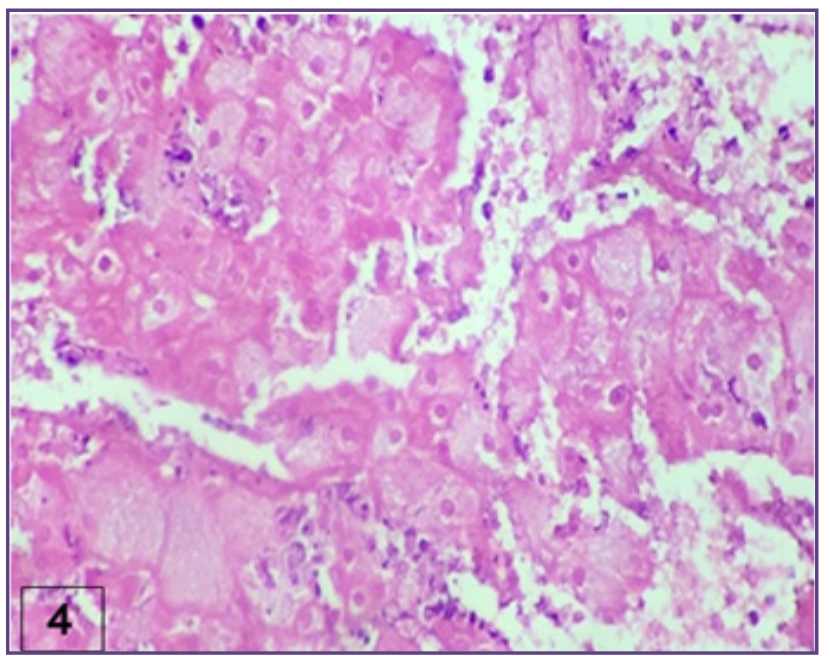

Fig. 4: Microphotograph showing round to polygonal cells with round, irregular hyperchromatic nucleus, peinuclear halo and abundant amount of granular eosinophilic cytoplasm (H\&E 400X).

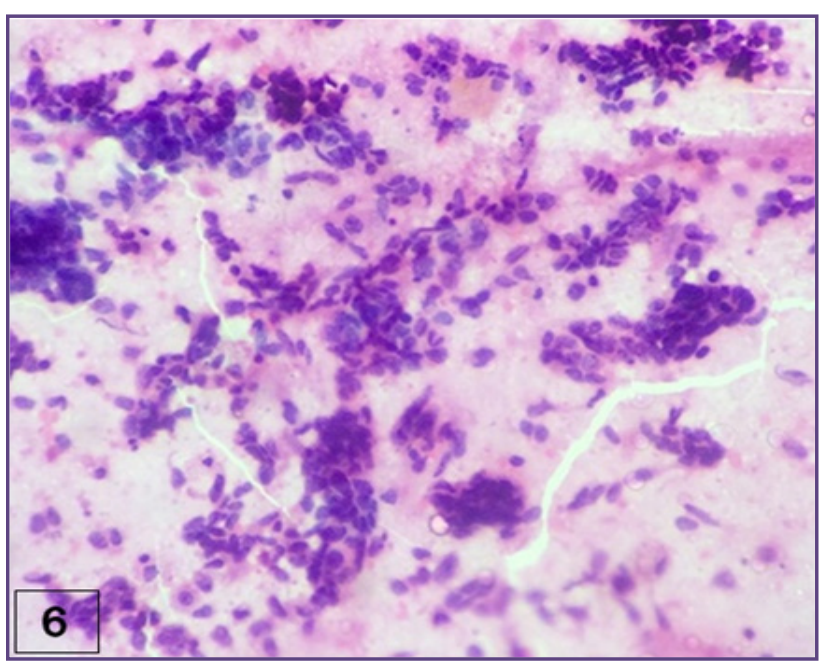

Fig. 6: Microphotograph of imprint smear showing blastemal component and few spindle cells (Giemsa 200X)

has a clean background. Further, the cells in clear cell RCC have indistinctcell borders and friable cytoplasm. ${ }^{[6,8]}$ Wilms tumor (Nephroblastoma) is the commonest renal neoplasm found in chidren accounting for $20 \%$ of all malignant tumors in this age group. ${ }^{[9]}$ It arises from the metanephric blastema and primarily occurs in infants and about $50 \%$ of cases occur before the age of 3 years. ${ }^{[9,10]}$

Cytomorphology shows triphasic pattern of cells comprising of blastemal, epithelial and mesenchymal stromal elements. Blastemal component is composed of small undifferentiated cells having scant cytoplasm. ${ }^{[9]}$ The epithelial component show ill formed tubules, glomeruli like structures which resembles the appearance of metanephric tubules and 
glomeruli respectively. ${ }^{[9]}$ The mesenchymal spindle shaped cells have fibroblastic configuration. ${ }^{[9]}$

Smears with predominance of blastemal cells could be mistaken for neuroblastoma, but the rosettes of neuroblastoma are pseudo rosettes which are multilayered containing central pink fibrillary material suggesting neuropil. ${ }^{[1]}$ Further neuroblastoma is NSE positive and $\mathrm{CK}$ negative in contrast to Wilms tumor. ${ }^{[9]}$ Other differential diagnoses for Wilms tumor include nonHodgkin lymphoma (NHL), rhabdomyosarcoma (RMS), Ewings sarcoma, desmoplastic small round cell tumor and other pediatric renal tumors like cystic nephroma, mesoblastic nephroma, rhabdoid tumor and clear cell sarcoma ${ }^{[9,11]} \mathrm{NHL}$ presents as monomorphic population of tumor cells without forming clusters and showing LCA positivity. Lymphoglandular bodies may be seen in the background. ${ }^{[9,11]}$ RMS show strap cells having cross striations. Further, the triphasic pattern and localization of the tumor to kidney favoursWilms tumor. ${ }^{[9,11]}$

Chandrakar et al did a study to evaluate the relevance of TIC with histopathological correlation in 110 cases. Out of 110 cases, four cases were of the kidney lesion which included two RCC and two cases of Wilms tumor. Among the four lesions, three cases correlated with histopathological diagnosis and the remaining one case (Wilms tumor) was unsatisfactory on imprint smear. ${ }^{[1]}$ Harnish B et al in their study on TIC comprising of 119 cases included a single case of transitional cell carcinoma of renal pelvis which was of non-diagnostic quality on imprint smear. ${ }^{[3]}$

\section{Conclusion}

TIC is a simple, rapid and inexpensive technique aiding in the early management. Renal tumors have distinct cytological features that facilitate diagnosis on touch imprint cytology. Although the major disadvantage of TIC is the failure to provide information on depth of invasion, early diagnosis helps in patients with high clinical suspicion and where there is indication for preoperative chemotherapy and radiotherapy.The knowledge and experience of the cytopathologist, skillfulness of the technician and size of the tumor are important variables that influence the results of TIC.

\section{References}

1. Chandrakar J, Srivastava S. Evaluation of the relevance of touch imprint cytology in the diagnosis of various neoplastic lesions. Int J Res Med Sci 2015;3:3046-50.

2. Kamatchi V, Babu AN, Sankari LS, Rajesh E. Imprint cytology. J Pharm BioallSci 2015;7:S207-8.

3. Harnish B, Nidhi V, Neena D. Usefulness of touch Imprint Cytology in Cancer diagnosis: A study of 119 cases. Int Res J Medical Sci 2014;2:19-25.

4. Ranjan A, Chandoke RK, Chauhan N, Kumari R. Study of Tumors by Imprint Cytology. Indian Journal of Clinical Practice 2013;24:472-7.

5. Ng CS, Wood GC, Silverman MP, Tannir MN, Tamboli P, Sandler MC. Renal Cell Carcinoma: Diagnosis, Staging, and Surveillance. AJR 2008;191:1220-32.

6. Mamatha K, Arakeri SU, Patil G. Chromophobe variant of renal cell carcinoma masquarding as renal oncocytoma on cytology. Int J Pharm Bio Sci 2015;6:(B) 59-63.

7. Granter SR, Renshaw AA. Fine needle aspiration of chromophobe renal cell carcinoma. American cancer society 1997;81:122-8.

8. Lee W. Imprint cytology of the chromophobe renal cell carcinoma: correlation with the histological and ultrastructural features. J Cytol 2011;28:77-80.

9. Dorothy LF, Pooja S. Giant adult Nephroblastoma - A case report. International $\mathrm{J}$ of medical and applied sciences 2013;2:346-9.

10. Rajwanshi A. Cytology of soft tissue tumors: Malignant small round cell tumors. J cytol 2008;25:89-92.

11. Alam K, Prasad S, Maheshwari V, Aggarwal S, Chana RS. Diagnostic role of fine needle aspiration cytology in Wilms tumor. J Cytol 2007;24:134-36.

*Corresponding author:

Dr Ambica C, Post Graduate, Department of pathology, BLDEU's Shri B. M. Patil Medical College, Hospital and Research centre,

Vijayapur-586103. Karnataka,India.

Phone: +91 9986149480

Email: dr.ambica04@gmail.com

Date of Submission : 08.01.2017

Financial or other Competing Interests: None. 